\title{
Survival of Dynamic Hip Screw in Unstable Intertrochanteric Fractures
}

\author{
ThudukuchiRamanathanAshok ${ }^{1}$, GanesanGanesanRam ${ }^{2}$, \\ Balasugumar Thamodharan ${ }^{3}$, Suresh Perumal ${ }^{4}$ \\ ${ }_{1,2,3,4}$ (Department of Orthopaedics, Sri RamachandraUniversity, Chennai-116,Tamilnadu,INDIA)
}

\begin{abstract}
: 51 patients with unstable intertrochanteric fracture treated with sliding hip screw alone were selected in our retrospective and prospective study for a period of 28 months with an average follow up of minimum 4 months.Lateral femoral wall integrity was assessed in all patients radiologically prior and after surgery and tip apex distance was calculated following DHS fixation as described by Baumgartner et al. 5 out of 51 patients had screw cutout within six months of surgery. In our study, unacceptable TAD combined with loss of lateral femoral wall integrity is a definite indicator of DHS implant cutout. Lateral femoral wall fracture resulted in six times higher risk of a reoperation due to technical failure when gold standard method of sliding hip screw was used. Tip Apex Distance alone was not a reliable indicator for screw cut out.The simple treatment guideline should be if the lateral femoral wall or greater trochanter is fractured, the use of DHS implant must be guarded.
\end{abstract}

Keywords:Intertrochanteric fracture, lateralfemoralwallintegrity $(L F W)$, zones in femoral head,tipapexdistance(TAD).

I. Introduction :

Intertrochanteric fracture is one of the most common factures of the hip especially in the elderly with porotic bones, usually due to low-energy trauma like simple falls.Problems of these fractures are (1) association with substantial morbidity and mortality (2) malunion (3) implant failure, cutout of screw head, and penetration into hip. (4) great financial burden to the family and (5) associated medical problem like diabetes, hypertension. It is universally agreed that the treatment of intertrochanteric fractures is stable internal fixation at the earliest opportunity. Stable fixation is the keystone for successful union of trochanteric fractures. Factors beyond the control of surgeon for successful treatment are: (i) fracture geometry and stability, (ii) bone quality, (iii) comminution. Factors under the control of surgeon are: (i) good reduction, (ii) proper choice of implant, (iii) proper surgical technique, and (iv) availability of modern operation rooms, entire set of implants, instrumentation and image intensifier.The factors most significant for instability and fixation failure are: (i) loss of posteromedial support, (ii) severe comminution, (iii) subtrochanteric extension of the fracture, (iv) reverse oblique fracture. (v) shattered lateral wall (vi) extension into femoral neck area and (vii) poor bone quality. Osteoporosis is particularly important in the fixation of proximal femoral fractures. The mechanism of failure has been the collapse of the neck-shaft angle into varus leading to cut out of the screw from the femoral head. There are various factors which results in the screw cut out, such as age of the patient, quality of the bone, pattern of the fracture, stability of reduction, angle of the implant and position of the lag screw. But there has been no clear consensus to the interrelationships or the relative importance of each factor. Most of the authors have recognized the importance of accurate placement of screw in the femoral head. There have been various methods to evaluate the position of the screw. We have used the method formulated by Baumgartner et al (JBJS Am,1995,77:1058-1064)- Tip apex distance and Lateral Femoral Wall Integrity by Palm et al JBJS (Am) 2007; $89: 470-475$.

\section{AIM :}

To analyze lateral femoral wall integrity and tip apex distance in unstable intertrochanteric fracture with DHS fixation an important predictor of screw cut out for reoperation.

\section{Materials And Methods :}

Our study was conducted at Sri Ramachandra Medical College and Hospital from June 2010 to October 2012 , it was prospective and retrospective study.We included all unstable intertrochanteric fractures fixed with DHS alone and excluded stable intertrochanteric fracture and intertrochanteric fractures treated with all other modalities. The major factors contributing to the fracture in our study group was person age, landing on the hip, inadequate reflexes and osteoporosis. AO/OTA classification was used to classify fracture pattern in all selected patient. 51 patients were selected totally among them male34(66.67\%),female17(33.33\%) and nature of injury 
was trivial fall-50(98\%) and RTA1(2\%). Majority of our patients are in the age group of 45-83yrs, with an average age of $64.67 \mathrm{yrs}$.According to AO/OTA classification fracture pattern of A2.1-22(43.13\%),A2.218(35.29\%)A2.3-5(9.8\%),A3.1-5(9.8\%),A3.2-1(1.96\%).Tip apex distance of Good limits $<25 \mathrm{~mm}-1(1.96 \%)$, acceptable limits (26-30mm)-11(21.56\%), poor limit (31-35mm)-19(37.25\%) and unacceptable limits $>35 \mathrm{~mm}$ $20(39.21 \%)$. In our study screws were most frequently placed in center-center zone (49\%) and least frequently posterior-inferior (1.96\%).Lateral femoral wall integrity preoperatively present-45(88.23\%), lost-6(11.76\%) and postoperatively present-22(43.13\%),lost-29(56.86\%).

\section{FIGURES AND TABLES :}

Table 1.Age distribution

\begin{tabular}{|c|c|}
\hline Age (In years) & No. of patients \\
\hline $21-30$ & - \\
\hline $31-40$ & - \\
\hline $41-50$ & $3(5.88 \%)$ \\
\hline $51-60$ & $19(37.25 \%)$ \\
\hline $61-70$ & $17(33.33 \%)$ \\
\hline $71-80$ & $8(15.68 \%)$ \\
\hline $81-90$ & $4(7.84 \%)$ \\
\hline
\end{tabular}

Table 2.Gender distribution

\begin{tabular}{|c|c|}
\hline Male & Female \\
\hline $34(66.77 \%)$ & $17(33.33 \%)$ \\
\hline
\end{tabular}

Table3.Mode of injury

\begin{tabular}{|c|c|}
\hline Trivial Fall & RTA \\
\hline $50(98 \%)$ & $1(2 \%)$ \\
\hline
\end{tabular}

Table 4.AO/OTA classification for intertrochanteric fracture

\begin{tabular}{|c|c|}
\hline AO/OTA- 31 & NOS. \\
\hline A 2.1 & $22(43.13 \%)$ \\
\hline A 2.2 & $18(35.29 \%)$ \\
\hline A 2.3 & $5(9.8 \%)$ \\
\hline A 3.1 & $5(9.8 \%)$ \\
\hline A 3.2 & $1(1.96 \%)$ \\
\hline A 3.3 & - \\
\hline
\end{tabular}

Table 5.Tip apex distance (TAD)

\begin{tabular}{|c|c|}
\hline TAD & Nos. \\
\hline Good $(<25 \mathrm{~mm})$ & $1(1.96 \%)$ \\
\hline Acceptable(26-30mm) & $11(21.56 \%)$ \\
\hline Poor $(31-35 \mathrm{~mm})$ & $19(37.25 \%)$ \\
\hline Unacceptable $(>35 \mathrm{~mm})$ & $20(39.21 \%)$ \\
\hline
\end{tabular}

Fig 1.Zones of Screw placement in femoral head

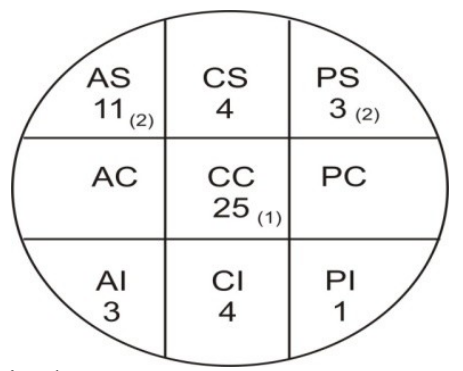

(No) - Numbers indicate screw cut out in that zone.

Fig 2.Lateral femoral wall integrity (LFW) 


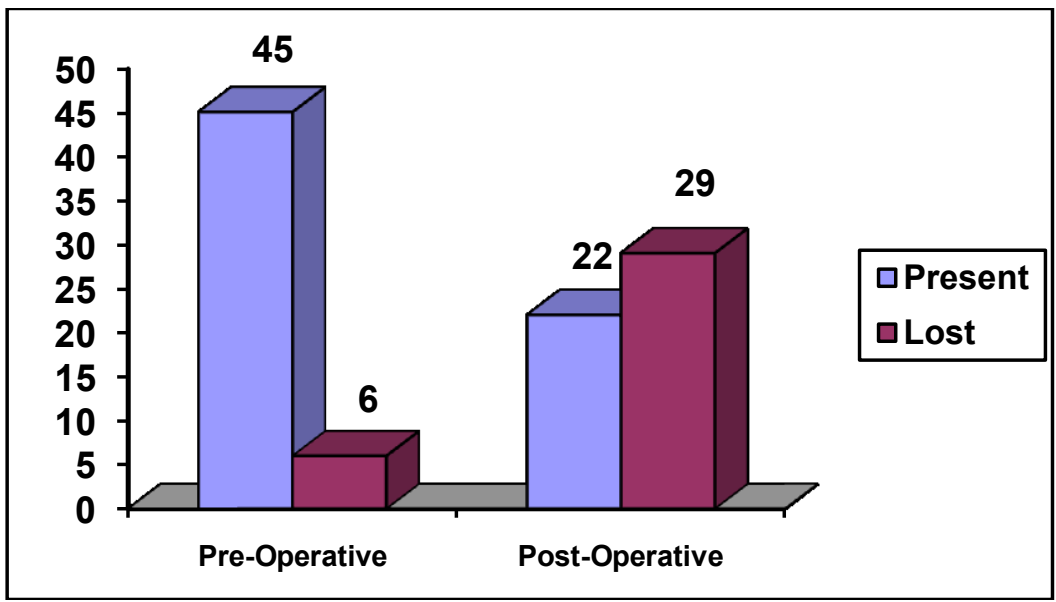

Table 6.Post opeative- Lateral femoral wall (LFW) integrity loss

\begin{tabular}{|c|c|}
\hline AO/OTA & Nos. \\
\hline A 2.1 & $9(40.90 \%)$ \\
\hline A 2.2 & $10(45.45 \%)$ \\
\hline A 2.3 & $4(18.18 \%)$ \\
\hline
\end{tabular}

\section{Results :}

1)In our study, 5 out 51 patients had screw cutout which occurred within six months after the surgery.2) In the study,sliding screw were most frequently placed in center-center zone $(49 \%)$ and least frequently in posterior-inferior $(1.96 \%)$ zone. 3)The highest rate of screw cutout occurred in anterior-superior(two of five screws) and posterior-superior(two of five screws), the rate of cutout in these two peripheral zones was significantly higher than rate in other zones. However, the placement of screws in any of the other zones had no predictive significance with respect to cut out.4) In five patients who had screw cutout TAD averaged $35.04 \mathrm{~mm}$ (range 30.4 to $38.8 \mathrm{~mm}$ ) compared with $34.91 \mathrm{~mm}$ (range, 24 to $46.5 \mathrm{~mm}$ ) in those who had no screw cutout.5) In our study lateral femoral wall was lost preoperatively (fracture pattern) in 6 patients $(11.76 \%)$ and intact in 45 patients $(88.23 \%)$, postoperatively loss of lateral femoral wall integrity was seen in 22 patients $(48.88 \%)$ we found that there was seven times higher risk of losing lateral wall integrity due to a technical failure when the gold standard method of sliding compression hip-screw fixation was used.

\section{Discussion :}

In our study we had used the AO/OTA classification and noticed that forty five fractures $(88.23 \%)$ of the 51 were type 31-A2 and six (12.77\%) were of type A31-3. In our study lateral femoral wall integrity (LFW) was lost preoperatively (fracture pattern) in 6 patients $(11.76 \%)$ and intact in 45 patients $(88.23 \%)$, postoperatively loss of lateral femoral wall integrity was seen in 22 patients $(48.88 \%)$.we found that there was seven times higher risk of losing lateral wall integrity due to a technical failure when the gold standard method of sliding compression hip-screw fixation was used. Twenty three patients out of twenty nine fractures (79.31\%) that were identified postoperatively, known to have occurred during the surgery itself had been classified as type A2.1 were 9 fractures (39.1\%), type A2.2 were 10 fractures $(43.42 \%)$ and type A2.3 were 4 fractures (17.39\%). In our study the tip apex distance (TAD) averaged 34.92mm (range, 24 to 46.50 ) for all 51 fractures. The screw cut out through the femoral head was noticed in 5 of the 51 patients. All the screw cut out occurred within 6 months after the surgery.In the 5 patients who had screw cut out TAD averaged $35.04 \mathrm{~mm}$ (range, 30.4 to $38.8 \mathrm{~mm}$ ) compared with $34.91 \mathrm{~mm}$ (range, 24 to $46.5 \mathrm{~mm}$ ) in those from which the screw had not cut-out. Forty five patients out of fifty one had sustained type A2.1, A2.2, A2.3 fracture and $80 \%$ ( 4 of the 5) of type A2.3 had an intraoperative fracture of the lateral wall compared with $40 \%$ ( 9 of the 22) of type A2.1. In our study screws were most frequently placed in center-center zone (49\%) and least frequently posterior-inferior $(1.96 \%)$. The highest rate of cutout occurred in the anterior-superior (two of five screw) and posterior-superior (two of five screw zones). The rate of cut out in these two peripheral zones was significantly higher than the rate in the center-centerzone.However, the placement of screws in any of the other four zones - that is, placement of $23.52 \%$ of all screws - had no predictive significance with respect to cut out.In our study one patient with type 31- A2.1 fracture and TAD $30.4 \mathrm{~mm}$ with lag screw in the center-center zone had screw cut out as the lateral femoral wall integrity was lost postoperatively. The other three patients in whom the screw had cutout were type 31A2.1,A2.2 fractures, but the lateral femoral wall integrity was lost postoperatively and the $\mathrm{TAD}$ was in the range of poor and unacceptable limits $(33.2-38.6 \mathrm{~mm})$. 


\section{Conclusion:}

1)In our study, unacceptable TAD combined with loss of lateral femoral wall integrity is a definite indicator of DHS implant cutout.

2)Lateral femoral wall fracture resulted in six times higher risk of a reoperation due to technical failure when gold standard method of sliding hip screw was used.

3)DHS has to be better avoided in AO/OTA $31 \mathrm{~A} 2.2$ and 31A2.3 intertrochantric fractures as the incidence of post operative loss of femoral wall integrity was statistically significant.

4)Tip Apex Distance alone was not a reliable indicator for screw cut out.

5)The simple treatment guideline should be if the lateral femoral wall or greater trochanter is fractured, the use of sliding hip screw must be guarded.

\section{References :}

[1]. Integrity of the Lateral Femoral Wall in Intertrochanteric Hip Fractures : An Important Predictor of a Reoperation. Henrik Palm. JBJS Am. 2007; $89: 470-475$

[2]. The value of the tip-apex distance in predicting failure of fixation of peritrochanteric fractures of the hip. MR Baumgaertner. JBJS Am. 1995; $77:$ 1058-1064

[3]. Prediction of Fixation failure after sliding hip screw fixation. Pervez et al. Vowler S. Injury 2004 Oct.; 35(10) : 994-998.

[4]. Bannister, G.C., Gibson, A.G., Ackroyd C.E., Newman, J.H., The fixation and prognosis of trochanteric fractures. A randomized prospective controlled trial.Clin.Orthop. 254:242-246, 1990.

[5]. Bridle, S.H., Patel, A.D.; Bircher M., and Calvert P.T.: Fixation of intertrochanteric fractures of femur. A randomized prospective comparison of the Gamma nail and dynamic hip screw. J.B.J.S. 73-B(2):330-334,1991.

[6]. Clawson, D.K., Trochanteric fractures treated by sliding screw plate fixation method. J. Trauma, 4:737-752, 1964.

[7]. Davis, T.R.C.; Sher, J.L.; Horsman, A.; Simpson, M., Porter, B.B.; and Checketts, R.G.: Intertrochanteric femoral fractures. Mechanical failure after internal fixation J.B.J.S., 72-B(1) : 26-31, 1989

[8]. Doherty, J.H., and Lyden, J.P., Intertrochanteric fractures of hip treated with the hip compression screw. Analysis of problems.ClinOrthop., 141:184-187,1979.

[9]. Kaufer, H. : Mechanics of treatment of hip injuries. Clin.Orthop.146: 53-61, 1980.

[10]. Kyle R.F.; Gustilo, R.B., and Premer R.F. : Analysis of six hundred and twenty two intertrochanteric fractures. A retrospective and prospective study. J.B.J.S. 61-A:216-221, March 1979.

[11]. Laros, G.S. and Moore, J.F., Complication of fixation in intertrochanteric fractures. Clin.Orthop, 101:110-119,1974.

[12]. Larsson, S. Friberg S; and Hanson L : Trochanteric fractures. Influence of reduction and implant position on impaction and complication. Clin.Orthop.259:130-139,1990.

[13]. Mainds, C.C. and Newman R.J. : Implant failures in patients with fractures of proximal femur and treated with sliding screw device. Injury, $20: 98-109,1989$.

[14]. Mulholland R.C. and Gunn, D.R. Sliding Screw plate fixation of intertrochanteric femoral fractures. J. Trauma, 12: 581-591, 1972.

[15]. Parker, M.J. : Cutting out of dynamic hip screw related to its position. J.B.J.S., 74 - B(4) : 625, 1992.

[16]. Schumpelick W.; and Jantzen P.M. : A new principle in the treatment of trochanteric fractures of femur. J.B.J.s. 37-A : 693-698, July 1955.

[17]. Thomas, A.P., : Dynamic hips screws that fail, Injury, $22: 45-46,1991$.

[18]. Whitelaw, G.P.; Segal, D; Sanzone C.F; Ober, N.S. and Hadley N : Unstable intertrochanteric / subtrochanteric fractures of the femur. Clin.Orthop.252:238-245, 1990.

[19]. Wolfang, G.L., Bryant, M.H., and O’Neil J.P. : Treatment of intertrochanteric fractures of femur using sliding screw plate fixation. Clin.Orthop.163: 148-158,1992.

[20]. Dimon, J.H., Hughston J.C., Unstable trochanteric fractures of the hip. J.B.J.S. 49A, 400-450, 1967.

[21]. Sarmiento A. : The unstable intertrochanteric : Treatment with a valgus osteotomy \& 1 Nail plate. J.B.J.S. 52A (10) : 13091318,1970 .

[22]. Current concept review - Intertrochantric fractures.GS Kulkarni, Rajiv limaye, Miliandkulkarni, Sunil kulkarni. Indian journal of Orthopaedics, January 2006;Vol 40: No.1: p $16-23$. 